\title{
Voting Theory and Pairwise Comparison Matrices
}

*Note: Do not include the author(s) names and information as this document will be blind reviewed and they will be entered during proposal submission.

\begin{abstract}
Borda's rule is an election method which is substitute for majority voting. The method was designed by Borda to overcome a defect of majority voting. First, we introduce Borda's rule, in which each voter votes his/her preference order of alternatives, and each alternative obtains scores corresponding to its ranks. Next, we formulate the voting results as a pairwise comparison matrix, from which we show that there is a very close similarity between Borda's rule and eigenvector method in AHP.
\end{abstract}

Keywords: voting theory, Borda's rule, pairwise comparison matrix

\section{Introduction}

In democratic society, we adopt majority voting for social choices. Majority voting, however, does not reflect the social preference completely. Let us consider the example.

\begin{tabular}{|c|c|c|c|c|}
\hline Rank & 4 voters & 4 voters & 7 voters & 6 voters \\
\hline 1st & $X$ & $X$ & $Y$ & $Z$ \\
\hline 2nd & $Y$ & $Z$ & $Z$ & $Y$ \\
\hline 3rd & $Z$ & $Y$ & $X$ & $X$ \\
\hline
\end{tabular}

There are three alternatives $X, Y, Z$, and 21 voters. 4 voters think $X>Y>Z$, another 4 voters think $Z>X>Y, 7$ voters think $Y>Z>X$, and 6 voters think $Z>Y>X$. In majority voting, we look only 1 st row in the table, and we choose $X$ with 8 votes. But we look pairwise comparisons, $X$ loses all comparisons; 8 voters think $X>Y$ but 13 voters think $Y>X$, and 8 voters think $X>Z$ but 13 voters $Z>X$. This defect was first pointed out by Borda, and he provided Borda's rule to overcome the defect. We add scores of their ranks to alternatives. For example, the 1st, the 2 nd, and the 3 rd obtain 2 points, 1 points, and 0 points respectively. So the total score of $X$ is $16(=4$ voters $\times 2$ points + 4 voters $\times 2$ points +7 voters $\times 0$ points +6 voters $\times 0$ points $), Y$ is 24 , and $Z$ is 23 , and hence $Y$ is chosen by Borda's rule with its total score 24 .

\section{Literature Review}

In 1762, J.J.Rousseau described "Du Contract Social" (Of the Social Contract), and he studied procedures of decision-making suitable for social preference. This is the beginning of scientific studies of social choices. In 1784, Borda pointed out a defect of majority voting, and he introduced scoring rules that overcome the defect.

\section{Hypotheses/Objectives}

We consider the following situations in this reserach. There are $m$ alternatives $X_{1}, X_{2} \ldots, X_{m}$, and there are $n$ voters. Each voter has his/her preference ranking of alternatives. One alternative should be chosen reflecting to social preference with voters' large satisfaction. 


\section{Research Design/Methodology}

We compare Borda's rules and AHP's pairwise comparisons. We point out that we can represent the scheme of Borda's rule into AHP's pairwise comparison matrices.

\section{Data/Model Analysis}

In the Borda's rule, we can interpret the total score of an alternative as the total number of wins of pairwise comparisons. If a voter thinks an alternative is $i$ th rank in his/her preference, the alternative defeats $(m-i)$ alternatives in $(m-1)$ pairwise comparisons. It corresponds to the score of $i$ th rank, so the sum of scores for all voters of the alternative is the total number of wins of the alternative in all pairwise comparisons.

Based on this, we can arrange the results of pairwise comparisons into a matrix $A=\left(a_{i j}\right)$, from which we can derive total scores of Borda's rule. Let define $|i>j|$ as the number of voters thinking $X_{i}>X_{j}$, and define an element $a_{i j}$ be the ratio of $|i>j|$ to $|j>i|$, that is $a_{i j}=|i>j| /|j>i|$. Because $|i>j|+|j>i|=n$, we have $|i>j|=n$. $a_{i j} /\left(1+a_{i j}\right)$. Therefore, we have the relation between the number of wins of $X_{i}$ in all pairwise comparisons and Borda's score $n B(i)$, such as $\sum_{j \neq i}|i>j|=n \sum_{j \neq i} a_{i j} /(1+$ $\left.a_{i j}\right) \equiv n B(i)$, that is, $B(i)$ is the summation of $a_{i j} /\left(1+a_{i j}\right)$ of $i$ th row of the matrix $A$. Since the matrix $A$ is reciprocal $\left(a_{i j}=1 / a_{j i}\right)$, same as AHP's pairwise comparison matrices. AHP's weight estimation methods is able to apply to voting with a constraint: there are no pair $(i, j)$ which holds $|i>j|=0$ or $|j>i|=0$. So we can detect total scores of Borda's rule from AHP's pairwise comparison matrix. Moreover, we will provide a new simple weight estimation for AHP. The weight of an alternative $X_{i}$ is $B(i) / \sum_{j=1}^{m} B(j)$. The term $a_{i j} /\left(1+a_{i j}\right)$ is a pairwise proportion of the robust priority estimation proposed by Lipovetsky and Conklin 2002. We put new interpretation of the method, and we justify detecting priorities from the terms.

\section{Limitations}

We can apply AHP's estimation to voting, and can apply Borda's rule to AHP's weight estimation. These depends on an assumption that each voter has ranking of all alternatives and there is no cycle in his/her preference.

\section{Conclusions}

By numerical experiments, we can confirm that results of AHP's eigenvector method almost equals to results of Borda's rule. In Borda's rule, we do not select an alternative that loses all pairwise comparisons (Fishburn and Gehrlein 1976). They will provide new evaluation principal for eigenvector method.

\section{Key References}

Lipovetskey, S., and Conklin, M. (2002), Robust estimation of priorities in the AHP, European Journal of Operational Research, 137, pp.110-122.

Fishburn, P.C., and Gehrlein, W.V. (1976), Borda's Rule, Positional Voting, and Condorcet's Simple Majority Principle, Public Choice, Vol.28, pp.79-88. 